\title{
Electroacupuncture promotes neurological functional recovery via the retinoic acid signaling pathway in rats following cerebral ischemia-reperfusion injury
}

\author{
JIANGCONG HONG ${ }^{1,2}$, GUANGWEN WU ${ }^{1}$, YULONG ZOU ${ }^{2}$, JING TAO $^{3}$ and LIDIAN CHEN ${ }^{4}$ \\ ${ }^{1}$ Academy of Integrative Medicine, Fujian University of Traditional Chinese Medicine, Fuzhou 350122; \\ ${ }^{2}$ Subsidiary Rehabilitation Hospital of Fujian University of Traditional Chinese Medicine, Fuzhou 350003; \\ ${ }^{3}$ Subsidiary of Rehabilitation Medicine, Fujian University of Traditional Chinese Medicine, \\ Fuzhou 350003; ${ }^{4}$ Fujian University of Traditional Chinese Medicine, Fuzhou 350122, P.R. China
}

Received July 18,2012; Accepted September 18, 2012

DOI: $10.3892 /$ ijmm.2012.1166

\begin{abstract}
Neurogenesis is regulated by a number of signaling pathways, including the retinoic acid (RA) pathway, a key regulator of neurogenesis in the subventricular zone (SVZ) and hippocampus. Acupuncture has been used to treat neurological conditions and is known to potentially enhance cell proliferation in the neurogenic area (hippocampal dentate gyrus and the SVZ of the lateral ventricle walls) in pathological conditions, which is associated with improved brain function. However, whether or not the neuroprotective effects of electroacupuncture (EA) are mediated by the regulation of the RA signaling pathway remains to be determined. Using a transient middle cerebral artery occlusion model, in the present study we evaluated the effect of EA on the neurological functional recovery, infarction volume and investigated the underlying molecular mechanisms. Two hundred and sixteen SD rats were randomly divided into 3 groups: sham, model group (ischemic rats without EA stimulation) and EA group (ischemic rats with EA stimulation on ST36 and LI11). Behavioral deficits were detected with high-resolution digital analysis of 24-h home-cage video recordings. Infarct volume was determined by triphenyltetrazolium hydrochloride staining and the expression of RA mRNA and protein was measured using RT-PCR and western blotting, respectively. We found that EA decreased the infarct volume, promoted neurological functional recovery and increased the RA mRNA and protein expression, compared with the model group. Findings of this study suggest that promoting neuro-
\end{abstract}

Correspondence to: Dr Jing Tao, Subsidiary of Rehabilitation Medicine, Fujian University of Traditional Chinese Medicine, Fuzhou 350003, P.R. China

E-mail: taojing01@yahoo.com.cn

Dr Lidian Chen, Fujian University of Traditional Chinese Medicine, 1 Huatuo Road, Shangjie Minhou, Fuzhou 350122, P.R. China

E-mail: lidianchen87@yahoo.com.cn

Key words: electroacupuncture, neurological function, retinoic acid, cerebral ischemia-reperfusion injury logical functional recovery by modulating RA expression in the post-ischemic brain is one of the mechanisms by which EA can be effective in the treatment of ischemic stroke.

\section{Introduction}

Cerebral ischemia is considered a major cause of morbidity and mortality worldwide. Excitotoxicity is a major pathophysiological mechanism associated with stroke-induced inflammation and oxidative stress-associated disruption of the blood-brain barrier, resulting in enhancing neuronal cell death during ischemic stroke (1). Recombinant tissue plasminogen activator (t-PA) is the preferred treatment for acute ischemic stroke, however, due to a limited time window few patients are able to receive this therapy. Furthermore, t-PA treatment is likely to increase the risk of hemorrhage (2). Therefore, development of alternative therapies for ischemic stroke is important.

Mounting evidence indicates that potential neural stem/progenitor cells are present in various brain regions outside of the subventricular zone (SVZ) and subgranular zone of the hippocampal dentate gyrus $(3,4)$. Stroke-induced neurogenesis has also been demonstrated in the adult human brain, even in patients of advanced age (5-7), indicating potential involvement of neural stem cells (8) and neurogenesis-regulating factors (9). Various factors regulate neurogenesis during development. One developmental molecule of particular interest in this regard is retinoic acid (RA), which is involved in the normal development of the central nervous system (10) and is also critical in the adult brain $(11,12)$. RA expression and signaling continues in the postnatal and adult brain, stimulating neonatal SVZ and adult hippocampal neurogenesis (13-16). The hypothesis that RA is crucial in the transcriptional model after nerve injury is supported by indirect evidence regarding the regulation of trauma-related genes, by observations of retinoid receptors and binding proteins after nerve injury and by dorsal root ganglia, retinal and spinal cord explant cultures (17). Retinoid-binding proteins are expressed in the SVZ-olfactory bulb pathway and RA receptors persist in the olfactory bulb (18). Transplantation of neural cells obtained from RA-treated 
cynomolgus monkey embryonic stem cells successfully enhanced the motor function of hemiplegic mice with experimental brain injury (19).

Acupuncture has been used to treat neurologic conditions, and acupuncture reportedly enhances cell proliferation in the neurogenic area (hippocampal dentate gyrus and the SVZ of the lateral ventricle walls) in pathological conditions (20-22), which is associated with enhanced brain function $(23,24)$. The LI11 (Quchi) and ST36 (Zusanli) acupoints have been selected for brain injury rehabilitation. Electroacupuncture (EA) at these acupoints has the potential to stimulate cell proliferation and reduce brain injury $(20,21,25-27)$. However, the mechanisms underlying the effects of acupuncture are poorly understood. Thus, whether or not the neuroprotective effects of EA are mediated by regulation of the RA signaling pathway should be investigated.

To extend the clinical observations of the potential neuroprotective effect of EA and help to establish a scientific foundation for further research, the effect of EA on the infarct volume, neurological functional recovery, and the expression of RA mRNA and protein were examined in this study. EA was found to decrease the infarct volume, promote neurological functional recovery and regulate the expression of RA mRNA and protein. This finding suggests that promoting neurological functional recovery through modulating RA expression in the post-ischemic brain is one of the mechanisms by which EA can be effective in the treatment of ischemic stroke.

\section{Materials and methods}

Reagents. The Raldh1, Raldh 2 and $\beta$-actin primers were purchased from Sangon Biotech (Shanghai) Co., Ltd. (Shanghai, China). Raldh1 and Raldh2 antibodies, horseradish peroxidase (HRP)-conjugated secondary antibodies and the antibody against $\beta$-actin were obtained from Cell Signaling Technology, Inc. (Danvers, MA, USA). Any other chemicals used, unless otherwise stated, were obtained from Sigma Chemical Company (St. Louis, MO, USA).

Animals. Healthy and pathogen-free male Sprague-Dawley rats $(\mathrm{n}=216)$, weighing 220-280 g, were purchased from SLAC Laboratory Animal Co., Ltd., Shanghai, China (Laboratory Animal Use Certificate no. SCXK(SH)2007000509570) and raised in a sterile environment. The care and use of the laboratory animals complied with the Guidance Suggestions for the Care and Use of Laboratory Animals, enforced by the Ministry of Science and Technology, China (2006) (28).

Animal model of transient middle cerebral artery occlusion. Animals were housed in a 12-h reverse light-dark cycle and were provided with food and water ad libitum. Rats were anesthetized with $10 \%$ chloral hydrate $(3.5 \mathrm{ml} / \mathrm{kg}$ body weight). Body temperature was maintained using a $37^{\circ} \mathrm{C}$ waterrecirculating pad. Transient middle cerebral artery occlusion (tMCAO) was produced for 120 min using the external carotid artery insertion method as described previously (29). Briefly, the left common carotid artery was exposed through a midline incision, and the internal and external carotid arteries were separated. A $3.0-\mathrm{mm}$ nylon monofilament with a tip rounded by heat was placed in the external carotid artery and advanced through the internal carotid artery until resistance occurred. The monofilament was left in place for $120 \mathrm{~min}$ and then removed under anesthesia. Animals were observed until recovery from anesthesia. The sham group was treated in the same way, although tMCAO was not induced. This protocol produced infarcts involving the striatum and cortex, with a mortality rate of $\sim 30 \%$. The rats were subsequently randomized into 3 experimental groups: EA group ( $n=72)$, model group $(n=72)$ and sham group $(n=72)$. The rats were tested at $1,7,14$ and 28 days subsequent to tMCAO. Eighteen rats were decapitated at each time point in each experimental group, after the neurological function was tested. Six rats were used for triphenyltetrazolium hydrochloride (TTC) staining, 6 rats were used for western blotting (Raldh1 and Raldh2 protein measurement), and the remaining 6 were used for Raldh1 and Raldh2 mRNA measurement.

Treatment. In the EA group, EA was applied to acupoints for hemiplegia. The acupoint prescription included ST36 (Zusanli, $5 \mathrm{~mm}$ below the head of fibula under the knee joint and $2 \mathrm{~mm}$ lateral to the anterior tibial tubercle) and LI11 (Quchi, in the depression lateral to the anterior aspect of the radius joint of the forelimb) at a depth of $5 \mathrm{~mm}$ into the skin with a stainless needle measuring $0.25 \times 20 \mathrm{~mm}$ in length with a guide-tube for 20 min (Wujiang Shenli Medical \& Health Material Co., Ltd., Wujiang, China). Electric stimulation was generated using an electrical stimulator (Huatuo SDZ-II; Suzhou Medical Appliance Factory Co., Ltd., China) for $20 \mathrm{~min}$ and the stimulation parameter exhibited dispersedense waves of a frequency of $5 / 20 \mathrm{~Hz}(28.5 \mathrm{msec} / 15 \mathrm{msec}$ pulse duration) and a current density of 2-4 mA. Model and sham groups did not undergo any EA, which was administered once a day for a period of 4 weeks.

Behavioral testing. Neurological function deficits were evaluated using the HomeCageScan system at 1, 7, 14 and 28 days after tMCAO. Each rat was placed in a separate cage identical to its home-cage, with fresh bedding, food and water. A video camera (Sony DCR-HC62) was positioned perpendicular to the long axis of the cage so that the field of view included the entire length of the cage. The rats were acclimatized to their new environment for $1 \mathrm{~h}$ and activity was recorded for $24 \mathrm{~h}$ (12-hour light/dark cycle) under standard fluorescent lighting. The rats were then returned to their home-cages. The full length of each video was analyzed using HomeCageScan (version 3.0; Clever Systems, Inc.) for the quantification of rat motor deficits, specifically quantifying behaviors including grooming, feeding, walking and rearing. The sum of the time spent in each behavior was calculated, yielding an index of total activity.

Measurement of infarct volume. Infarct volume was assessed at 1, 7, 14 and 28 days after tMCAO using TTC staining. Animals were sacrificed by $10 \%$ chloral hydrate overdose, brains were rapidly removed, sectioned coronally at $2-\mathrm{mm}$ intervals from the frontal pole and immersed in TTC (2\%) at $37^{\circ} \mathrm{C}$ for $20 \mathrm{~min}$, followed by formaldehyde (4\%) for $15 \mathrm{~min}$. The hemispheric infarcted area in each section was calculated by subtracting the area of normal TTC-stained brain in the ipsilateral cortex from the contralateral area. The 
Table I. PT-PCR primers.

Gene

Primer sequence

Annealing temperature $\left({ }^{\circ} \mathrm{C}\right)$

\begin{tabular}{lll}
\hline Raldh1 & Forward 5'-CTTCTTTGTCCAGCCCACAGTCT-3' \\
& Reverse 5'-GTTCACCCAGTTCTCGTCCATTT-3' & 55 \\
Raldh2 & Forward 5'-GTCGTCAACATTCTGCCAGGGTAT-3' \\
& Reverse 5'-CTGCTCCACAGCGTAGTCCAAGTC-3' & 56 \\
$\beta$-actin & Forward 5'-ACTGGCATTGTGATGGACTC-3' \\
& Reverse 5'-CAGCACTGTGTTGGCATAGA-3' & 55 \\
\hline
\end{tabular}

data were analyzed with a computer-based image analysis system (Adobe Photoshop 8.0). The sum of the areas of all the sections was calculated and then multiplied by $2 \mathrm{~mm}$, yielding the volume.

RNA extraction and RT-PCR analysis. A total of 72 rats ( $n=6 /$ group) were sacrificed by decapitation at $1,7,14$ and 28 days after tMCAO. The ischemic zone of rat brain was rapidly removed and stored at $-80^{\circ} \mathrm{C}$ for subsequent analysis. Total RNA from brain tissue was isolated with TRIzol reagent (Invitrogen). Oligo (dT)-primed RNA $(1 \mu \mathrm{g})$ was reverse-transcribed with SuperScript II reverse transcriptase (Promega) according to the manufacturer's instructions. The obtained cDNA was used to determine the mRNA amount of Raldh1 or Raldh 2 by PCR with Taq DNA polymerase (Fermentas). $\beta$-actin was used as an internal model. The primers used in these reactions are listed in Table I. The amplified products were analyzed by $1.5 \%$ agarose gel electrophoresis. Optical density ratios for Raldh1, Raldh 2 and $\beta$-actin were used for the semi-quantitative analyses.

Western blot analysis. The ischemic zone of rat brain was sectioned, and the corresponding samples were homogenized with a homogenizer in lysis buffer (NP-40 lysis buffer and $100 \mathrm{mM}$ PMSF). The homogenates were centrifuged at $14,000 \mathrm{rpm}$ for $30 \mathrm{~min}$ at $4^{\circ} \mathrm{C}$. The supernatant was collected and the total protein content was determined using a Micro BCA protein assay kit with bovine serum albumin as the standard (Pierce Chemical, Rockford, IL, USA). Equal amounts of protein $(20 \mu \mathrm{g})$ were boiled in loading buffer and separated by $12 \%$ SDS-PAGE gel under reducing condition using $80 \mathrm{~V}$ for $1 \mathrm{~h}$. The proteins were then electrophoretically transferred onto nitrocellulose membranes using the iBlot Western Detection Stack/iBlot Dry Blotting system (Invitrogen). Membranes were blocked for $30 \mathrm{~min}$ with agitation at RT in SuperBlock T20 (TBS) blocking buffer (Thermo Scientific, Rockford, IL, USA). Membranes were washed in TBS with $0.25 \%$ Tween-20 (TBST) and exposed to primary antibodies against Raldh1 or Raldh2 (1:1,000) overnight at $4^{\circ} \mathrm{C}$ on a rocking platform or rotator. $\beta$-actin $(1: 1,000)$ was also measured as an internal control for protein loading. After membranes were washed in TBST, secondary horseradish peroxidase (HRP)-conjugated antibodies (anti-goat) were added at 1:5,000 dilution for $1 \mathrm{~h}$ at room temperature and the membranes were washed again in TBST. The antibody-bound protein bands were then detected with ECL, and images

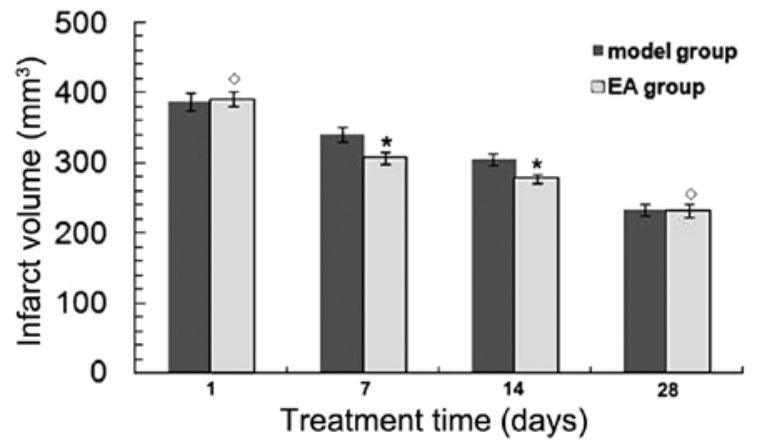

Figure 1. Effect of EA treatment on the infarct volume in rats following tMCAO. After treatment with or without EA for the indicated time periods, the infarct volume changes were determined by TTC staining. Data shown are averages with SD (error bars) from 6 individual rats in each group. ${ }^{*} \mathrm{P}<0.05,{ }^{* *} \mathrm{P}<0.01$ vs. the model group at 7 and 14 days. ${ }^{\circ} \mathrm{P}>0.05$ vs. the model groups at 1 and 28 days.

were captured using a Bio-Rad ChemiDoc XRS+ (Bio-Rad, Hercules, CA, USA). The ratio of gray scale values of the target protein to the internal control was used to measure the relative amount of Raldh1 and Raldh2.

Statistical analysis. Data are presented as the means \pm SD for the indicated number of independently performed experiments. Comparisons between multiple groups were conducted with one-way ANOVA, whereas within each group data were analyzed with analysis of intraclass variance analysis. Differences between the 2 groups were assessed using the Student's t-test. P-values of $<0.05$ and $<0.01$ were considered as significant and highly significant differences, respectively.

\section{Results}

$R A$ reduces infarct volume in rats following $t M C A O$. TTC staining was performed to determine the infarct volumes in brain sections at 1, 7, 14 and 28 days after tMCAO. The infarct volume was found to be decreased in a time-dependent manner in both the EA and model groups $(\mathrm{P}<0.05)$ (Fig. 1). At 7 and 14 days, the infarct volume was significantly decreased in the EA group $(\mathrm{P}<0.05)$ vs. the model group. No significant differences were detected in the infarct volume between the EA and model groups at 1 and 28 days after tMCAO (P>0.05), indicating that rats were able to recover naturally after tMCAO. 

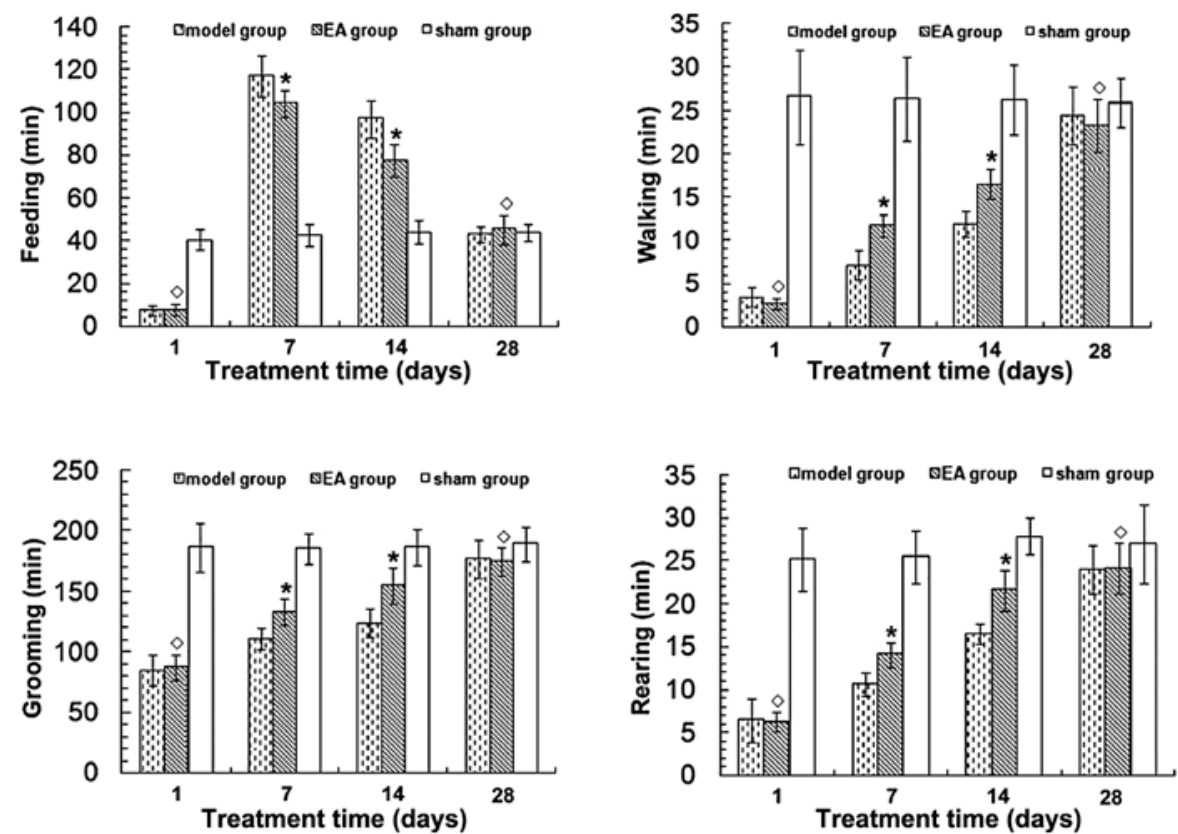

Figure 2. Effect of EA treatment on the neurological function in rats following tMCAO. After treatment with or without EA for the indicated time periods, the walking, rearing, feeding and grooming time changes were analyzed using a software program designed for rat home-cage behavioral assessment. Bar graphs represent the mean minutes of activity (sum of minutes spent walking, rearing, feeding and grooming, $\pm \mathrm{SD} ; 6$ individual rats in each group). ${ }^{*} \mathrm{P}<0.05,{ }^{* *} \mathrm{P}<0.01$, vs. the model group at 7 and 14 days. ${ }^{\circ} \mathrm{P}>0.05$ vs. the model groups at 1 and 28 days.

A

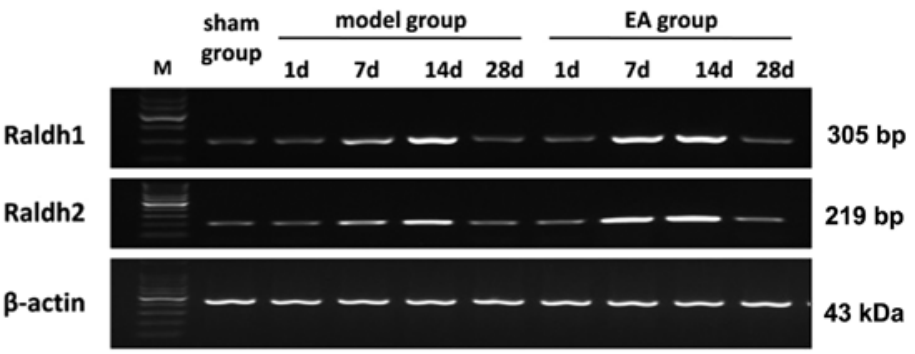

B

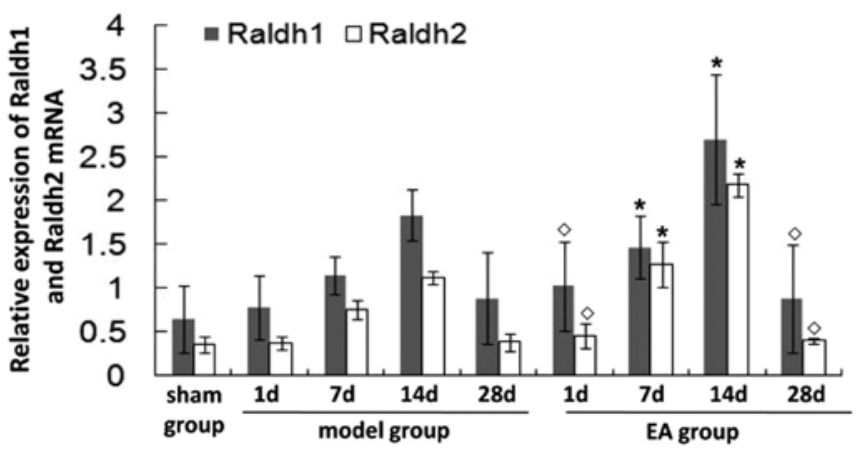

Figure 3. Effect of EA treatment on the mRNA expression of Raldh1 and Raldh2 in rats following tMCAO. (A) After treatment with or without EA for the indicated time periods, the ischemic zone of rat brain was selected from each group, and the mRNA expression levels of Raldh1 and Raldh2 were determined by RT-PCR. $\beta$-actin was used as the internal control for RT-PCR. For each sample, the RT-PCR analysis was performed in triplicate. Data shown are representatives. (B) Densitometric analysis. ${ }^{*} \mathrm{P}<0.05,{ }^{* *} \mathrm{P}<0.01$ vs. the model group at 7 and 14 days. ${ }^{\circ} \mathrm{P}>0.05$ vs. the model groups at 1 and 28 days.

RA improves neurological function in rats following tMCAO. Cerebral cortex infarction caused neurological function deficits in rats, mainly manifested as right forelimb paralysis. To quantify this deficit, 24-h video recordings were analyzed using a software program designed for rat home-cage behavioral assessment. Neurological function was found to have improved in a time-dependent manner in the EA and model groups $(\mathrm{P}<0.05)$ (Fig. 2). Time spent walking, rearing and grooming was increased, while time spent feeding was reduced in the EA group 7 and 14 days after tMCAO $(\mathrm{P}<0.05)$ compared with the model group. No significant differences in neurological function were observed between the EA and model groups at 1 and 28 days after tMCAO $(\mathrm{P}>0.05)$. No neurological function deficits were evident in the sham group. 
A

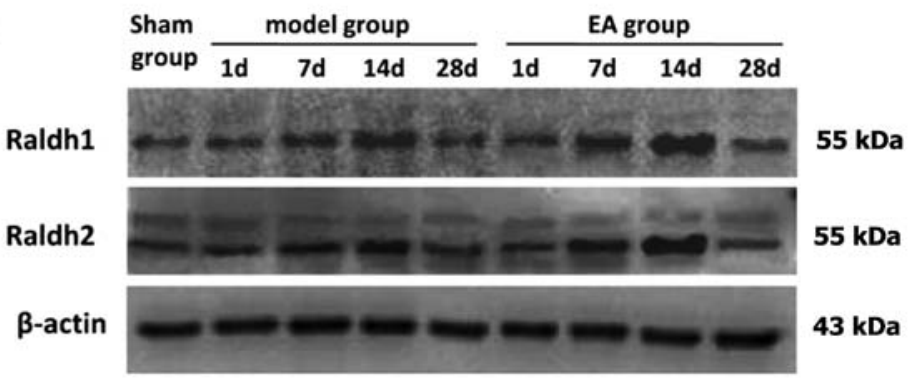

B

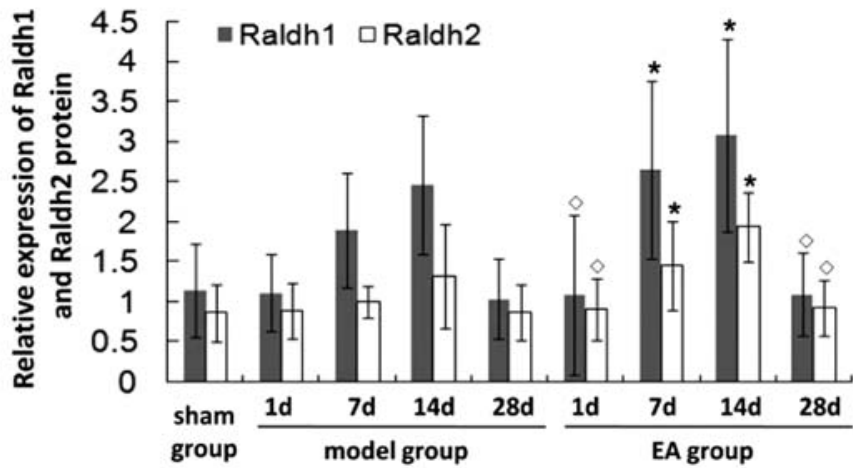

Figure 4. Effect of EA treatment on the protein expression of Raldh1 and Raldh2 in rats following tMCAO. (A) After treatment with or without EA, the ischemic zone of rat brain was selected from each group, and the protein expression levels of Raldh 1 and Raldh 2 were determined by western blotting. $\beta$-actin was used as the internal control for western blotting. For each sample, the western blot analysis was performed in triplicate. Data shown are representatives. (B) Densitometric analysis. ${ }^{*} \mathrm{P}<0.05,{ }^{* *} \mathrm{P}<0.01$, vs. the model group at 7 and 14 days. ${ }^{\circ} \mathrm{P}>0.05$ vs. the model groups at 1 and 28 days.

EA treatment regulates the expression of Raldh1 and Raldh2 after $t M C A O$. To explore the mechanism of the potential neuroprotective effect of EA, RT-PCR and western blot analysis were carried out to examine the mRNA and protein expression in the ischemic brain after tMCAO. In the model group, Raldh1 and Raldh2 mRNA was greatly increased at 7 days, peaked at 14 days and then gradually decreased (Fig. 3). Additionally, in the model group, higher Raldh1 and Raldh 2 mRNA levels were found at 7 and 14 days $(\mathrm{P}<0.05)$ compared with the sham group. In the EA group, the Raldh1 and Raldh 2 mRNA levels were higher than those in the model group at 7 and 14 days $(\mathrm{P}<0.05)$. No significant differences were detected in the Raldh1 and Raldh 2 mRNA expression levels between the EA and model groups at 1 and 28 days after tMCAO ( $\mathrm{P}>0.05)$, and the pattern of protein expression of Raldh1 and Raldh2 was similar to their respective mRNA levels (Fig. 4). These findings suggest that EA promotes neurological functional recovery in rats following tMCAO through the regulation of expression of the RA family.

\section{Discussion}

Neural stem cells from the adult forebrain SVZ give rise to olfactory bulb interneurons, while those in the hippocampal dentate gyrus generate new neurons in the granule cell layer (18). Brain insults such as seizure, stroke and trauma activate neural stem cells to proliferate more rapidly, migrate into injured regions and form new neurons and glia (30-36).

Retinoids, in particularly the active metabolite of vitamin A, RA, is known to stimulate neonatal SVZ and adult hippocampal neurogenesis (14-16). Retinol and its derivatives exert their biological actions via specific nuclear receptors
(RARs and RXRs) that regulate gene transcription (37). RA receptors also interact with other nuclear receptors that possess neuroprotective properties, such as 1,25-dihydroxyvitamin D (9) that is neuroprotective against stroke (38) and RXR receptors that form heterodimers with the vitamin D3 receptor (RXR-VDR). RXR is also able to form a dimer with thyroid hormone receptors, and thyroid hormone derivatives are protective against infarction (39). Thus, the neuroprotective effects of retinol and RA may be mediated, at least in part, by their formation of heterodimers with other nuclear receptors. In addition to its effect on nuclear receptors, retinol exerts acute effects on other potentially protective biological sites including calcium channels and gap junction channels, as well as having antioxidant properties. Calcium channel antagonists and antioxidants protect the brain from ischemia in animal models of stroke (40). RA also induced the expression of midkine, which is protective against brain ischemia (41).

RA is synthesized from retinol in a two-step pathway involving oxidation first to retinaldehyde, then to RA. The former step is predominantly catalyzed by retinol dehydrogenases, the latter step by retinaldehyde dehydrogenases (Raldhs), which are encoded by Raldh1, Raldh2 and Raldh3 expressed in non-overlapping patterns. Raldh3 is expressed only in the eye and facial region/ventral retina and the ganglionic eminence of the telencephalon. Later in development, this enzyme can be detected by zymography in a variety of sites, including the liver and adult skin. Raldh1 is present in only the dorsal retina, ventral mesencephalon and projections from here to the corpus striatum and the otic vesicle regions (data not shown). Although Raldh2 is absent from most of the brain proper, it is strongly expressed in the leptomeninges that 
surround it (42). In the present study, we found that the Raldh1 and Raldh2 mRNA levels of the EA and model groups were greatly increased at 7 days, peaked at 14 days and gradually decreased at 28 days after tMCAO. A marked increase was observed in the Raldh1 and Raldh 2 mRNA levels at 7 and 14 days in the EA group $(\mathrm{P}<0.05)$ compared with the model group. Expression of the Raldh1 and Raldh2 protein was similar to that of Raldh1 and Raldh2 mRNA.

Behavior is known to be the ultimate and most complex output of the brain. To quantify neurological function deficit, 24-h video recordings were conducted and digitally analyzed. The assessment of locomotor deficits using this method has been established in models of Huntington's and prion disease (43), with a similar approach having been described recently in a rat $\mathrm{ICH}$ model (44). Compared with traditional methods of behavioral assessment in stroke models, this digital approach has a number of benefits (45-47). First, it requires minimal investigator participation, reducing labor costs and the potential for bias. Second, it facilitates the analysis of night-time behavior. The validity of daytime testing of nocturnal animals in stroke models has never been established and is questionable given the behavioral inhibition and cognitive disturbances observed in rats during light phase testing. Third, this digital approach measures behaviors that are directly relevant to the animal's functional status. Most importantly, however, it eliminates the variability inherent in measuring brief episodes of behavior in animals stressed by environmental variation and human interference.

In this study, we found that time spent walking, rearing and grooming was markedly increased, whereas time spent feeding was reduced with locomotor activity improvement in the EA group at 7 and 14 days after tMCAO, compared with that in the model group $(\mathrm{P}<0.05)$. The changes we found in the neurological function were concomitant with improvements in RA mRNA and protein expression. These results therefore suggest that the administration of EA is likely to promote neurological functional recovery and regulate the expression of RA mRNA and protein. To the best of our knowledge, this is the first study to report that EA regulates RA expression.

It was also demonstrated that there was no significant difference in the infarct volume, neurological function, expression of RA mRNA and protein between the EA and model groups at 28 days after tMCAO $(\mathrm{P}>0.05)$, indicating that rats were able to recover naturally after tMCAO. Spontaneous recovery may be due to neurite regeneration and synapse remodeling, as well as a reduction in brain edema. A series of plasticity responses after tMCAO, including the release of neurotrophic factors, synthesis of neuron-specific proteins and rise of synaptic excitation, is involved in the process of functional recovery (48). Additionally, our study examined the correlation between neurogenesis and the use of young rats in behavioral recovery. The findings of this study are in contrast with the reality that the elderly population is most often affected by strokes and these people, unlike young healthy rodents, also have a multiplicity of other disease co-morbidities, such as hypertension and diabetes that along with increased age, are likely to limit or alter the neurogenic response post-stroke. Indeed, aged animals exhibit stroke-induced neurogenesis, however, their response is 'muted' compared with young animals $(49,50)$. Although the spontaneous recovery of neurological function deficit may occur after tMCAO, the administration of EA significantly promoted neural function recovery and decreased infarct volume. Raldh1 and Raldh2 mRNA and protein expression was increased by EA treatment following tMCAO compared to the model group.

In conclusion, administration of EA decreased the infarct volume, promoted neurological functional recovery and demonstrated that this neuroprotective effect is attributed, at least in part, to the expression of RA mRNA and protein. These findings likely provide an experimental basis for the treatment of ischemic stroke by electroacupuncture.

\section{Acknowledgements}

This study was supported by the National Natural Science Foundation of China (no. 30901935) and the Key Laboratory for Orthopedics and Sports Rehabilitation of the Ministry of Education, China.

\section{References}

1. Lo EH, Dalkara T and Moskowitz MA: Mechanisms, challenges and opportunities in stroke. Nat Rev Neurosci 4: 399-415, 2003.

2. Madden K: Optimal timing of thrombolytic therapy in acute ischaemic stroke. CNS Drugs 16: 213-218, 2002.

3. Willaime-Morawek S and van der Kooy D: Cortex- and striatum-derived neural stem cells produce distinct progeny in the olfactory bulb and striatum. Eur J Neurosci 27: 2354-2362, 2008.

4. Nakagomi T, Taguchi A, Fujimori Y, Saino O, Nakano-Doi A, Kubo S, Gotoh A, Soma T, Yoshikawa H, Nishizaki T, Nakagomi N, Stern DM and Matsuyama T: Isolation and characterization of neural stem/progenitor cells from post-stroke cerebral cortex in mice. Eur J Neurosci 29: 1842-1852, 2009.

5. Jin K, Wang X, Xie L, Mao XO, Zhu W, Wang Y, Shen J, Mao Y, Banwait $S$ and Greenberg DA: Evidence for stroke-induced neurogenesis in the human brain. Proc Natl Acad Sci USA 103: 13198-13202, 2006.

6. Macas J, Nern C, Plate KH and Momma S: Increased generation of neuronal progenitors after ischemic injury in the aged adult human forebrain. J Neurosci 26: 13114-13119, 2006.

7. Minger SL, Ekonomou A, Carta EM, Chinoy A, Perry RH and Ballard CG: Endogenous neurogenesis in the human brain following cerebral infarction. Regen Med 2: 69-74, 2007.

8. Landgren $\mathrm{H}$ and Curtis MA: Locating and labeling neural stem cells in the brain. J Cell Physiol 226: 1-7, 2011.

9. Hodge RD and Hevner RF: Expression and actions of transcription factors in adult hippocampal neurogenesis. Dev Neurobiol 71: 680-689, 2011.

10. Ross SA, McCaffery PJ, Drager UC and De Luca LM: Retinoids in embryonal development. Physiol Rev 80: 1021-1054, 2000.

11. McCaffery P, Zhang J and Crandall JE: Retinoic acid signaling and function in the adult hippocampus. J Neurobiol 66: 780-791, 2006.

12. Werner EA and Deluca HF: Retinoic acid is detected at relatively high levels in the CNS of adult rats. Am J Physiol Endocrinol Metab 282: E672-E678, 2002.

13. Calza L, Giuliani A, Fernandez M, Pirondi S, D'Intino G, Aloe L and Giardino L: Neural stem cells and cholinergic neurons: regulation by immunolesion and treatment with mitogens, retinoic acid, and nerve growth factor. Proc Natl Acad Sci USA 100: 7325-7330, 2003.

14. Haskell GT and LaMantia AS: Retinoic acid signaling identifies a distinct precursor population in the developing and adult forebrain. J Neurosci 25: 7636-7647, 2005.

15. Wang TW, Zhang $\mathrm{H}$ and Parent JM: Retinoic acid regulates postnatal neurogenesis in the murine subventricular zoneolfactory bulb pathway. Development 132: 2721-2732, 2005.

16. Jacobs S, Lie DC, DeCicco KL, Shi Y, DeLuca LM, Gage FH and Evans RM: Retinoic acid is required early during adult neurogenesis in the dentate gyrus. Proc Natl Acad Sci USA 103: 3902-3907, 2006.

17. Mey $\mathbf{J}$ and McCaffery P: Retinoic acid signaling in the nervous system of adult vertebrates. Neuroscientist 10: 409-421, 2004. 
18. Plane JM, Whitney JT, Schallert T and Parent JM: Retinoic acid and environmental enrichment alter subventricular zone and striatal neurogenesis after stroke. Exp Neurol 214: 125-134, 2008.

19. Ikeda R, Kurokawa MS, Chiba S, Yoshikawa H, Ide M, Tadokoro M, Nito S, Nakatsuji N, Kondoh Y, Nagata K, Hashimoto T and Suzuki N: Transplantation of neural cells derived from retinoic acid-treated cynomolgus monkey embryonic stem cells successfully improved motor function of hemiplegic mice with experimental brain injury. Neurobiol Dis 20: 38-48, 2005

20. Kim EH, Jang MH, Shin MC, Lim BV, Kim HB, Kim YJ, Chung JH and Kim CJ: Acupuncture increases cell proliferation and neuropeptide $\mathrm{Y}$ expression in dentate gyrus of streptozotocin-induced diabetic rats. Neurosci Lett 327: 33-36, 2002.

21. Kim EH, Kim YJ, Lee HJ, Huh Y, Chung JH, Seo JC, Kang JE Lee HJ, Yim SV and Kim CJ: Acupuncture increases cell proliferation in dentate gyrus after transient global ischemia in gerbils. Neurosci Lett 297: 21-24, 2001.

22. Liu Q, Yu J, Mi WL, Mao-Ying QL, Yang R, Wang YQ and Wu GC: Electroacupuncture attenuates the decrease of hippocampal progenitor cell proliferation in the adult rats exposed to chronic unpredictable stress. Life Sci 81: 1489-1495, 2007.

23. Cheng H, Yu J, Jiang Z, Zhang X, Liu C, Peng Y, Chen F, Qu Y, Jia Y, Tian Q, Xiao C, Chu Q, Nie K, Kan B, Hu X and Han J: Acupuncture improves cognitive deficits and regulates the brain cell proliferation of SAMP8 mice. Neurosci Lett 432: 111-116, 2008.

24. Naylor AS, Bull C, Nilsson MK, Zhu C, Bjork-Eriksson T, Eriksson PS, Blomgren K and Kuhn HG: Voluntary running rescues adult hippocampal neurogenesis after irradiation of the young mouse brain. Proc Natl Acad Sci USA 105: 14632-14637, 2008 .

25. Yun SJ, Park HJ, Yeom MJ, Hahm DH, Lee HJ and Lee EH: Effect of electroacupuncture on the stress-induced changes in brain-derived neurotrophic factor expression in rat hippocampus. Neurosci Lett 318: 85-88, 2002.

26. Gao J, Wang S, Wang X and Zhu C: Electroacupuncture enhances cell proliferation and neuronal differentiation in young rat brains. Neurol Sci 32: 369-374, 2011.

27. Jang MH, Shin MC, Lee TH, Lim BV, Shin MS, Min BI, Kim H, Cho S, Kim EH and Kim CJ: Acupuncture suppresses ischemia-induced increase in c-Fos expression and apoptosis in the hippocampal CA1 region in gerbils. Neurosci Lett 347: 5-8, 2003.

28. The Ministry of Science and Technology of the People's Republic of China: Guidance Suggestions for the Care and Use of Laboratory Animals. 2006-09-30.

29. Longa EZ, Weinstein PR, Carlson S and Cummins R: Reversible middle cerebral artery occlusion without craniectomy in rats. Stroke 20: 84-91, 1989.

30. Arvidsson A, Collin T, Kirik D, Kokaia Z and Lindvall O Neuronal replacement from endogenous precursors in the adult brain after stroke. Nat Med 8: 963-970, 2002

31. Goings GE, Sahni V and Szele FG: Migration patterns of subventricular zone cells in adult mice change after cerebral cortex injury. Brain Res 996: 213-226, 2004.

32. Lichtenwalner RJ and Parent JM: Adult neurogenesis and the ischemic forebrain. J Cereb Blood Flow Metab 26: 1-20, 2006.

33. Parent JM, Valentin VV and Lowenstein DH: Prolonged seizures increase proliferating neuroblasts in the adult rat subventricular zone-olfactory bulb pathway. J Neurosci 22: 3174-3188, 2002.
34. Parent JM, Vexler ZS, Gong C, Derugin N and Ferriero DM: Rat forebrain neurogenesis and striatal neuron replacement after focal stroke. Ann Neurol 52: 802-813, 2002.

35. Parent JM, Yu TW, Leibowitz RT, Geschwind DH, Sloviter RS and Lowenstein DH: Dentate granule cell neurogenesis is increased by seizures and contributes to aberrant network reorganization in the adult rat hippocampus. J Neurosci 17: 3727-3738, 1997.

36. Zhang RL, Zhang ZG, Zhang L and Chopp M: Proliferation and differentiation of progenitor cells in the cortex and the subventricular zone in the adult rat after focal cerebral ischemia. Neuroscience 105: 33-41, 2001.

37. Liden $\mathrm{M}$ and Eriksson U: Understanding retinol metabolism: structure and function of retinol dehydrogenases. J Biol Chem 281: 13001-13004, 2006.

38. Wang Y, Chiang YH, Su TP, Hayashi T, Morales M, Hoffer BJ and Lin SZ: Vitamin D(3) attenuates cortical infarction induced by middle cerebral arterial ligation in rats. Neuropharmacology 39: 873-880, 2000.

39. Doyle KP, Suchland KL, Ciesielski TM, Lessov NS, Grandy DK, Scanlan TS and Stenzel-Poore MP: Novel thyroxine derivatives, thyronamine and 3-iodothyronamine, induce transient hypothermia and marked neuroprotection against stroke injury. Stroke 38: 2569-2576, 2007.

40. Sato Y, Meller R, Yang T, Taki W and Simon RP: Stereo-selective neuroprotection against stroke with vitamin A derivatives. Brain Res 1241: 188-192, 2008.

41. Harvey BK, Shen H, Chen GJ, Yoshida Y and Wang Y: Midkine and retinoic acid reduce cerebral infarction induced by middle cerebral artery ligation in rats. Neurosci Lett 369: 138-141, 2004

42. McCaffery P and Drager UC: Regulation of retinoic acid signaling in the embryonic nervous system: a master differentiation factor. Cytokine Growth Factor Rev 11: 233-249, 2000.

43. Steele AD, Jackson WS, King OD and Lindquist S: The power of automated high-resolution behavior analysis revealed by its application to mouse models of Huntington's and prion diseases. Proc Natl Acad Sci USA 104: 1983-1988, 2007.

44. Otero L, Zurita M, Aguayo C, Bonilla C, Rodríguez A and Vaquero J: Video-Tracking-Box linked to Smart software as a tool for evaluation of locomotor activity and orientation in brain injured rats. J Neurosci Methods 188: 53-57, 2010.

45. Roedel A, Storch C, Holsboer F and Ohl F: Effects of light or dark phase testing on behavioural and cognitive performance in DBA mice. Lab Anim 40: 371-381, 2006.

46. Spruijt BM and DeVisser L: Advanced behavioural screening: automated home cage ethology. Drug Discov Today Technol 3: 231-237, 2006.

47. Chen L, Zhang X, Chen-Roetling J and Regan RF: Increased striatal injury and behavioral deficits after intracerebral hemorrhage in hemopexin knockout mice. J Neurosurg 114: 1159-1167, 2011

48. Stichel CC and Muller HW: Experimental strategies to promote axonal regeneration after traumatic central nervous system injury. Prog Neurobiol 56: 119-148, 1998.

49. Gao P, Shen F, Gabriel RA, Law D, Yang E, Yang GY, Young WL and SU H: Attenuation of brain response to vascular endothelial growth factor-mediated angiogenesis and neurogenesis in aged mice. Stroke 40: 3596-3600, 2009.

50. Leasure JL and Grider M: The effect of mild post-stroke exercise on reactive neurogenesis and recovery of somatosensation in aged rats. Exp Neurol 226: 58-67, 2010. 